\title{
LeRC Rail Accelerators: Test Designs and Diagnostic Techniques
}

L. M. Zana, W. R. Kerslake, J. C. Sturman,

S. Y. Wang, and F. F. Terdan

Lewis Research Center

Cleveland, Ohio

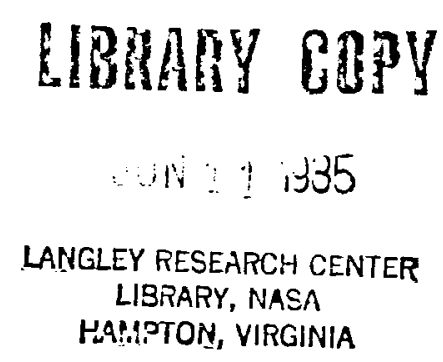

Prepared for the

Second Symposium on Electromagnetic Launch Technology sponsored by the Institute of Electrical and Electronics Engineers

Cambridge, Massachusetts, October 11-14, 1983 


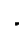




\title{
LERC RAIL ACCELERATORS: TEST DESIGNS AND DIAGNOSTIC TECHNIQUES
}

\author{
by L. M. Zana, W. R. Kerslake, J. C. Sturman, \\ S. Y. Wang, and F. F. Terdan \\ National Aeronautics and Space Administration \\ Lewis Research Center \\ Cleveland, Ohio 44135
}

\section{Abstract}

As a part of electromagnetic propulsion research at Lewis Research Center (LeRC), an in-house effort has been initiated to investigate the feasibility of using rail accelerators for various in-space and to-space propulsion applications. A 1 meter, $4 \times 6 \mathrm{~mm}$ bore accelerator has been designed with the goal of demonstrating projectile velocities of $15 \mathrm{~km} / \mathrm{sec}$ using a peak current of $200 \mathrm{kA}$. A second rail accelerator, 1 meter long with a $12.5 \times 12.5 \mathrm{~mm}$ bore, has been designed with clear polycarbonate sidewalls to permit visual observation of the plasma arc.

This paper presents a study of available diagnostic techniques and their application to the rail accelerator. Specific topics of discussion include the use of interferometry and spectroscopy to examine the plasma armature as well as the use of optical sensors to measure rail displacement during acceleration. Standard diagnostics such as current and voltage measurements will also be discussed.

\section{Introduction}

NASA space propulsion mission studies have examined the use of rail accelerators in launching ton-size payloads directly from the earth's surface to space. Rice, et al.1 assessed the potential feasibility and benefits of a conceptual Earth-to-Space Rail Launcher (ESRL) system capable of deep-space nuclear waste disposal and earth-to-orbit launch applications. The development of this concept included system requirements and definition, safety, environmental impact, costs, and technology status. The preliminary assessment indicated that such mission applications are not only technically feasible but also environmentally and economically beneficial. An in-house research program at LeRC has been initiated to investigate the technical feasbility of rail accelerators. The emphas is of the program is directed toward determining whether any limiting mechanisms exist which would prevent projectiles from reaching hypervelocities ( $>15 \mathrm{~km} / \mathrm{sec}$ ) or prevent scaling to large bore accelerators. Characterization of the plasma armature is considered to be of high importance in achieving such determinations.

The first portion of this paper briefly describes the LeRC rail accelerator facility. A detailed description of the two distinct rail accelerator types to be tested will be given along with design considerations. The first accelerator is 1 meter long with a $4 \times 6 \mathrm{~mm}$ bore designed for improved strength at high currents $(\sim 200 \mathrm{kA})$. The second accelerator is 1 meter long with a $12.5 \times 12.5 \mathrm{~mm}$ bore. It has clear polycarbonate (Lexguard) side walls to permit observation of the plasma arc and projectile acceleration along the entire length.

A major portion of this paper presents a study of available diagnostic techniques and their application to rail accelerator experiments. Characterization of the plasma armature involves electron number density and temperature measurements. These measurements present a difficult problem as densities are expected to be in the $10^{19} / \mathrm{cm}^{3}-10^{20} / \mathrm{cm}^{3}$ range with temperatures around $5 \times 10^{4} \mathrm{~K}$. Theoretically, at such high densities only blackbody radiation should be observed, but experimentalists ${ }^{2}$ have found it possible to get transmission data. A survey of available diagnostic methods indicates that a Mach-Zehnder interferometric technique may be applicable to calculate electron number density. Our approach will be to look at the spectrum over the widest wavelength range possible using spectroscopy. An optical multichannel analyzer will be employed for this purpose. A standard tungsten ribbon lamp will be used for intensity calibration. other diagnostic techniques to be discussed include the use of high speed framing and streak camera photography to investigate arc formation and acceleration, in addition to transient electrical measurements. Static and transient pressure measurements will be attempted through the use of optical sensors.

Mr. David Bauer and Dr. John Barber provided experimental data and took part in many helpful conversations concerning rail accelerator experiments. $\mathrm{Mr}$. Charles J. Michels and Dr. Alan Larson each conducted a seminar on available plasma armature diagnostic techniques.

\section{Description of Experiments}

\section{Rail Accelerator Operation}

The basic railgun configuration consists of two parallel conductors bounding an electrically insulated projectile. See Fig. 1. A conduct ive armature (whether solid or plasma) is placed behind the projectile. Current passing along one rail, across the armature, and returning through the other rail will generate a magnetic field. The interaction of the current with the field between the rails produces a force which accelerates the projectile. This force is given by

$$
F=\frac{1}{2} L I^{2}
$$

where $L^{\prime}$ is the inductance per unit length of the accelerator and $I$ is the current.

It is apparent, then that high acceleration requires an effective accelerator design (high L') matched to a high current energy source.

One method of operating a rail accelerator involves the charging of an inductive energy store from a capacitor bank with subsequent switching of the energy (current) into the accelerator. An appropriately sized inductor acts as a low impedance current source and produces nearly uniform projectile acceleration. 4 Figure 2 shows the electrical schematic for the circuit. A closing switch (crowbar) placed across the bank will prevent ringing of the circuit, i.e., huge current and voltage reversals. The charging cycle of an inductor can be relatively long, sometimes on the order of several hundred microseconds; however, the switching of the energy into the gun must be done as quickly as possible. In cases such as this, it is often advisable to use a high current opening switch (commutating fuse) across the breech of the accelerator. The fuse, sized to vaporize at peak circuit current, serves as a low resistance conductor during the inductor charging time, thus preventing premature acceleration of the projectile. 5,6 


\section{Rail Accelerator Facility}

Figure $3(a)$ displays the LeRC rail accelerator test facility. Energy storage is provided by four capacitor modulues charged from a single power supply. Each module consists of six capacitors with individual firing ignitions and is capable of delivering $60 \mathrm{~K}$ Joules of energy at $10 \mathrm{kV}$ (Fig. 3(b)). Each module is rated for an output of $200 \mathrm{kA}$ but circuit load impedances limit the current to $125 \mathrm{kA}$ or less. A complete description of the LeRC electromagnetic propulsion research facility is given in Ref. 7 , along with details of the design and operation of the pulsed energy system.

The output of the modules is coaxially connected to the center of a pyramidal-shaped copper header assembly. The purpose of the assembly is to provide equal resistance paths for the module outputs as the current is distributed from the center to the outer plates of the header. A $12.5 \mu \mathrm{H}$ inductor is connected to the header assembly to shape the capacitor output pulse. However, the inductor can be shorted out of the circuit to give higher currents with faster times to peak. A commutating fuse is not necessary when the coil is shorted, as the projectile will accelerate only a few centimeters during the first quarter cycle. Current from the inductor passes through a coaxial feed-thru into the vacuum test chamber. In order to minimize circuit resistance, cool-amping (silver plating) of all copper joints was done during assembly. Also, Noalox joint compound was put on all Al-Al and Al-Cu connections to prevent the formation of a nonconductive Aluminum-0xide layer.

The test chamber is designed sectionally and can accommodate rail accelerators up to 10 meters in length. The chamber al lows for testing in pressures from atmospheric to $10^{-4} \mathrm{Atm}$. The charge/discharge cycle of the bank is controlied by a console in an observation room adjacent to the test facility. All major electronics (oscilloscopes, etc.) are also housed in this adjacent room.

\section{Rail Accelerator Designs}

One of the first research efforts of LeRC in rail accelerator technology was an experimental program to investigate the performance of a single-stage, high velocity rail accelerator. Designed and built by IAP Research, Inc., the 3 meter long, $4 \times 6 \mathrm{~mm}$ bore accelerator was designed for launch velocities exceeding $10 \mathrm{~km} / \mathrm{sec}$. Bore size was dictated by energy storage capability. A series of experiments was conducted at Lawrence Livermore National Laboratory (LLNL) using a $375 \mathrm{~kJ}$ capacitor bank. Figure 4 shows a cross section of the simple accelerator design. The bore was defined by two $6 \mathrm{~mm}$ square copper rails, groove-fit into a fiber glass reinforced epoxy (G-10) insulating structure. The G-10 grooves were undercut at the inner corners to reduce stress.

Phenolic compression plates of $2.5 \mathrm{~cm}$ thickness and stainless steel bolts clamped the insulation structure together. Inductance bridge measurements ranging from 0.5 to $20 \mathrm{kHz}$ showed that the effective inductance per unit length ( $L^{\prime}$ ) of the railgun approached the high frequency limit ${ }^{8}$ of $0.5 \mu \mathrm{H} / \mathrm{m}$.

The projectiles used were made of polycarbonate and weighed approximately 0.21 grams. A polyethylene obturator was placed behind the projectile to prevent plasma leakage around the projectile during acceleration. A piece of copper foil behind the obturator served to initiate the plasma armature.

Analys is of magnetic probe $(\mathrm{dB} / \mathrm{dt})$ data revealed that the projectile only accelerated for a fraction of the total rail length. One hypothes is for the low performance is the development of secondary current paths due to the transition of a copper oxide layer on the launcher bore to a highly conductive state. A de- tailed presentation of this theory as well as a description of the LLNL test series is given in Refs. 5 and 6 .

Another possible explanation for the lack of continuous acceleration, however, may $l i e$ in a material failure of the G-10 structure pieces. G-10 has good mechanical strength properties in the planes perpendicular to the fiber layers, however, the parallelto-f iber mechanical properties of G-10 are very poor. Inspection of the insulation structure revealed progressive crack formation between the glass fiber planes and then final delamination of the G-10 pieces at peak current levels of 110-136 kA. The combination of the outward rail forces and the notch sensitivity in the groove corners may have permitted delamination and subsequent pressure force leakage during the acceleration process. The presence of a black sooty carbon deposit on the layers of delamination gives evidence to pressure leakage. A lack of this carbon deposit on the mating G-10 surfaces seems to indicate that the material failure occurred before, and not as a result of, the pressure leakage.

\section{One Meter Long $-4 \times 6 \mathrm{~mm}$ Bore Accelerator}

The first accelerator to be tested in the LeRC facility is a shorter, redesigned version of the above. The purpose of these tests is to verify the mechanical strength of the redesigned accelerator for peak currents up to $200 \mathrm{kA}$. The improved design will then be extended to longer lengths (up to $10 \mathrm{~m}$ ) in the hope of reaching the high velocities.

Figure 5 shows a cross section of the accelerator. one meter long sections of $\mathrm{G}-10$, in good condition from the LLNL experiments, were remachined for this assembly. The bore is stil1 $3.85 \times 6.35 \mathrm{~mm}$ but it is defined by two separate, inner structure pieces which hold the rails. The inner pieces sit in the outer G-10 structure such that the $p$ lane of the rails is oriented in a direction perpendicular to the direction of the outer fiber layers. Each inner piece is round $f$ it to the outer structure for additional stress relief.

With this design, the clamping action of the steel bolts is now against the outward rail forces. Distance from the steel bolts to the rails, however, is reduced and a small loss in ' $L^{\prime}$ is expected. New phenolic compression plates have been fabricated for this accelerator; the thickness of the plates has been increased to $3.75 \mathrm{~cm}$ to increase stiffness.

One Mete: Long - $12.5 \times 12 \mathrm{~mm}$ Bore Accelerator

A second accelerator has been fabricated to permit observation of plasma arc acceleration. Figure 6 shows a cross section of the assembly.

The rails are $12.5 \times 18.5 \mathrm{~mm}$ half-hard copper bars machined to a $0.02 \mathrm{~mm}$ tolerance. The configuration is rail-over-rail against the bolt clamping action. The rails are seated between two cross-shaped lense pieces which define a $12.5 \mathrm{~mm}$ square bore. G-10 is used as the outer structure; again, the orientation of the glass fiber layers is perpendicular to the major loading stresses.

The lense material is a general purpose, clear polycarbonate (Lexguard ${ }^{\$}$ ). It has a laminated, marresistant surface and machines easily in 1 meter sections so that the bore length is uninterrupted. Lexguard also has excellent impact and tensile strength properties. Its index of refraction $(n=1.586)$ is close to that of quartz.

Phenolic compression plates clamp the entire structure; the bolts are torqued to $13.5 \mathrm{~N} \cdot \mathrm{m}$ $(10 \mathrm{ft} 1 \mathrm{~b})$. In this accelerator design, the top and bottom G-10 structure pieces do not meet. The $12.5 \mathrm{~mm}$ gap allows for the placement of diagnostic equipment directly against the lense. 


\section{Projectiles}

The projectiles are polycarbonate rectangular blocks, machined to dimensions about $0.02 \mathrm{~mm}$ smaller than the bore size. The projectile for the $4 \times 6 \mathrm{~mm}$ accelerator weighs approximately $0.2 \mathrm{~g}$ while the projectile for the $12.5 \times 12.5 \mathrm{~mm}$ accelerator weighs $2.3 \mathrm{~g}$. In both cases, the plasma armature is generated with a small piece of aluminum foil ( 0.4 mil thick): no obturator is used. A precision air gauge measures the bore dimensions along the entire length to an accuracy of $\pm 0.01 \mathrm{~mm}$.

\section{Commutating Fuse}

For efficient energy transfer, the commutating fuse must be "matched" to the circuit so that interruption occurs at peak current and the capacitor bank discharges completely during the first quarter pulse. The fuse package is illustrated in Fig. 7(a). It consists of a Copper wire (approx. $11 \mathrm{~cm}$ long) mounted coaxially in a $2.5 \mathrm{~cm}$ diameter copper tube. The wire and tube conduct current across the breech of the accelerator while the inductor is being charged. The current flowing through the wire generates more heat than can be lost to the surroundings. When the wire vaporizes, or "bursts," due to this heat input, the circuit opens. Similar fuses were designed and used in the LLNL rail accelerator tests conducted earlier. 5,6

Tucker and $\operatorname{Toth}^{9}$ developed a computer code for the prediction of the behavior of exploding wires of various elements. The proper wire cross-sectional area needed to meet the matching criteria is given by

$$
A=\left[\frac{1}{9} \int i^{2} d t\right]^{1 / 2}
$$

where

A is the cross sectional area in $\mathrm{mm}^{2}$

$i$ is the current in Amps

and

$\mathrm{g}$ is the specific action constant in $\mathrm{A}^{2} \mathrm{sec} / \mathrm{mm}^{4}$

The copper tube is packed with a fine grade glass reinforcement filler (sand) to contain the wire explosion and prevent restrike. The brass plugs on the ends of the fuse wire serve as a heat sink at the connection points. For high level tests, two or more fuses can be used in parallel.

\section{Fuse Holder}

In order to ease current commutation into the accelerator once the wire has vaporized, the fuse package must be located as close to the breech as possible to minimize inductance. The fuse holder, shown in Fig. 7(b), serves several purposes. Firstly, it provides both a mechanical and electrical connection of the rail accelerator to the coaxial feed-thru. It is also capable of holding up to three parallel fuses (depending upon the energy level requirements of the test).

The fuse holder mounts easily on the rails extending from the accelerator assembly. The two rails lie adjacent to aluminum plates which clamp to the coaxial feed-thru. These connectors dog-leg to the feed-thru so that the gun assembly is oriented railover-rail in the test chamber. This configuration is necessary to permit easy access to side port diagnostics.

A $2.54 \mathrm{~cm}$ thick piece of $\mathrm{G}-10$ insulation provides a supporting base for the fuse holder. The rails and coaxial connectors are sandwiched between this supporting base and two aluminum angle bars which hold the three fuses. Bakelite cylinders were fabricated for use in cases where less than three fuses are needed (or in the low circuit inductance case where no commutating fuse is needed).

A centerpiece $(G-10)$ insulates the bore gap area between the rails. It extends outward $0.6 \mathrm{~cm}$ in all directions to prevent arc-over at the assembly edges. A counterbored $G-10$ piece sits atop the angle bars to prevent arcing also.

The entire fuse holder assembly is held together

with $1.25 \mathrm{~cm}$ diameter stainless steel bolts torqued to $27 \mathrm{~N} \cdot \mathrm{m}(20 \mathrm{ft} \cdot 1 \mathrm{~b})$. Brass shims placed under the clamping surfaces to assure a tight fit. The holder was designed so that it could be adapted for use with both rail accelerators with a minimal exchange of parts.

\section{Muzzle Suppressor}

In some rail accelerator tests the energy left in the system after the projectile has exited the muzzle is extremely high, sometimes as much as $100 \mathrm{~kJ}$. In order to prevent or minimize damage to local component parts of the accelerator, a muzzle suppressor has been fabricated to provide a current path for the excess energy once the arc has reached the muzzle.

Figure 8 displays a side view of the suppressor. It consists of two $2.5 \mathrm{~cm}$ thick stainless steel bars clamped in an aluminum-boron nitride assembly. Boron nitride was chosen as the insulation material because of its high temperature capability. The assembly is mounted on the accelerator phenolic structure, angling off so that it does not interfere with the projectile free-flight or any diagnostic equipment.

The suppressor is designed so that it adds no resistance or inductance to the system during the acceleration process. When the arc reaches the muzzle, it will transfer across the tapered gap between the rails and the stainless steel bars, dissipating the remaining system energy. The bars of the muzzle suppressor are separated by $3 \mathrm{~mm}(1 / 2$ of the rail gap to encourage the continuation of the rail arc). The bars are of sufficient mass to absorb the excess energy and to remain below $500^{\circ} \mathrm{C}$.

Figures 9(a) and (b) display top and side views of the 1 meter, $4 \times 6 \mathrm{~mm}$ bore rail accelerator assembly. The fuse holder and muzzle suppressor are also shown.

\section{Plasma Diagnostic Techniques}

\section{Theoretical Models of the Plasma Armature}

The plasma armature makes an excellent driving mechanism for the projectile because it has low mass, makes good electrical contact with the rails, and is conductive at the high current levels required. Characterization of the plasma arc is necessary to assess and predict the hydrodynamic and electromagnetic forces generated in the accelerator. Also, accelerator performance can be directly related to dynamic properties of the arc such as conductivity, heat transfer, and stability.

Theoretical groundwork in the modeling of the plasma arc was done by McNab. 10 Powell and Batteh 11 extended this model with a detailed fluid-mechanical and electrodynamic analysis. Assuming a steady-state solution, major flow variables such as pressure, electron number density, and temperature were found to vary nonlinearly with position in the arc. Powell12 later extended this model to 2-dimensions. A computer simulation code developed by Thiol3 also exists which models and predicts the physical properties of a quasistatic plasma armature.

Using the scaling relations developed in Ref. 11, estimates of the range of plasma parameters to be expected were made. For an acceleration current of 
$6-30 \mathrm{kA} / \mathrm{mm}$ of rail height, electron number densities are expected to be in the $1019-10^{20} / \mathrm{cm}^{3}$ range with temperatures around $10^{4} \mathrm{~K}$. The electric and magnetic field strengths at the center of the arc are estimated at $600-6200 \mathrm{~V} / \mathrm{m}$ and $5-20$ Tesla, respectively. The pressure may be as high as $400 \times 10^{6} \mathrm{~N} / \mathrm{m}^{2}$ at the Teading edge of the arc.

Such high values, particularly number density and temperature, severely limit the kinds of diagnostic techniques that can be used for plasma characterization. Theoretically, at such high densities and temperatures only blackbody radiation should be observed but experimentalists have found it possible to get some transmission data.

\section{Interferometry Density Measurement}

Optical interferometry is a well-established technique for the measurement of electron number density and distribution. It measures the refractivity of a material by comparing the phase of the wavefront of interest (i.e., through the plasma arc) with that of a reference wavefront. The refractive index of the plasma is proportional to the number density of the electrons. 14

Figure 10 gives a simplified description of a Mach-Zehnder interferometer. It consists of four mirrors, labeled $A, B, C$ and $D$ in the figure. Mirrors $A$ and $D$ are half-silvered beam splitters while mirrors $B$ and $C$ are totally reflecting. A beam from a light source (usually a laser) is expanded with a telescope and then is sent longitudinally through the plasma. A reference beam travels through a compensation structure below. The recombined wavefronts of the two beams produces an interference fringe pattern on the $f i l m$. This pattern is determined by the optical path difference between the two arms of the interferometer. A narrow filter is used to block out any stray light or plasma self-luminosity before reaching the camera lens.

Assuming that neutral gas density effects can be neglected, the phase shift caused by the plasma is given, in units of fringes, as

$$
N=\int \frac{(\mu-1)}{\lambda} d 1
$$

where

$N$ is the number of fringes

$\mu$ is the electron index of refraction

1 is plasma length

and

$\lambda$ is the wavelength

For the general case of an ionized plasma in the presence of an external magnetic field, the resulting dispersion relation gives an equation for the index of refraction

$$
\mu=\left[1-\frac{w_{p}^{2}}{w^{2}\left(1 \pm w_{e} / w\right)[1+i(v / w)]}\right]^{1 / 2}
$$

where

$w_{p} \equiv$ plasma frequency $=\left(\frac{4 \pi n_{e} e^{2}}{m_{e}}\right)^{1 / 2}$

$\nu \equiv$ frequency of collisions that a typical electron makes with heavier particles

$\mathrm{w}_{\mathrm{e}} \equiv$ electron cyclotron frequency

and

$w=2 \pi c / \lambda$
Assuming $w_{p}, w_{e}$, and $v \ll w, E q$. (3) can be rewritten as

$$
N=-\frac{1}{2 \pi} \frac{e^{2} \lambda}{m_{e} c^{2}} \int n_{e} d 1
$$

\section{where}

e is the electron charge

$m_{e}$ is the electron mass

$\mathrm{n}_{\mathrm{e}}$ is electron number density

and

$c$ is the speed of light

The Mach-Zehnder interferometer has the attractive feature that the test and reference beams are widely separated in space, which is convenient in avoiding obstructions of the rail accelerator structure. Also, the test beam only needs to make one pass through the plasma. Results are easy to interpret and no calibration technique is necessary.

The interferometer, however, is difficult to construct and requires precise positioning of the mirrors. Also, for good resolution, all mirrors and windows must be optically flat to $\lambda / 10$ and be able to pass the desired wavelength. Another limitation on the usefulness of an interferometric technique is the effect of bending the light beam caused by the presence of appreciable transverse density gradients in the plasma. The deflection of the traveling light rays, if severe, will result in an overlapping of the fringe pattern. A Schlieron or shadowgraph method can be used to detect these gradient variations. 15

In applying the Mach-Zehnder interferometer to the rail accelerator, the laser test beam must first traverse the lexguard windows. When unpolarized light is reflected from a plane surface, e.g., that separating air and the lexguard, the reflected light is partially polarized depending upon the angle of incidence and the ratio of indexes of refraction of the two media. At a special angle of incidence, the reflected light will be completely polarized; however, the transmitted light will only be partially polarized because only a small fraction of the incident beam is reflected. The angle at which this phenomenon occurs is given by Brewster's law

$$
\tan \theta_{B}=\frac{n_{2}}{n_{1}}
$$

where $n_{1}$ is the refractive index of the first medium (air) and $n_{2}$ is the refractive index of the second medium (glass).

For the railgun application, the angle $\theta$ at which the glass must be positioned with respect to the incident laser beam is

$$
{ }^{\theta} B=\tan ^{-1}\left(\frac{1.586}{1.0}\right)=57.8^{\circ}
$$

\section{Spectroscopic Temperature Measurements}

Before obtaining transmission data, a spectroscopic technique will be used to survey the entire plasma spectrum. The advantages of spectroscopy are that it does not disturb the plasma to be investigated and that a large amount of information can be interpreted from a calibrated spectrum. Figure 11 illustrates the spectral, or specific, intensity of one line. Plasma temperature, or the lower limit of plasma temperature, may be obtained from the maximum intensity while the corresponding wavelength, $\lambda_{0}$, gives information on the state of ionization of a certain element. The line profile itself contains 
information on the kinetic temperature of the emitter or the density of the perturbor. Further, electron number density can often be determined from the continuous background. 16

Local Thermal Equillibrium (LTE) denotes a state which does not differ much from that of an ideal system in that all energy distributions, with the exception of radiation energy, obey ideal equillibrium relationships. For a plasma in LTE, the fraction of atoms in a particular excited state is given by the Boltzmann formula as

$$
\frac{n_{i}}{n}=\frac{g_{i} e^{-E_{i} / k T}}{Z(T)}
$$

where

$n_{\mathfrak{i}}$ - population in quantum state $\mathbf{i}$

$n$ - total population of identical atoms

$g_{j}$ - statistical weight or degeneracy

$E_{i}$ - excitation energy of the ith state

$T$ - absolute temperature

and

$Z(T) \equiv \sum_{i} g_{i} e^{-E_{i} / k T}$, partition function

Further, the relative population between two different energy levels $i$ and $k$, is described by

$$
\frac{n_{i}}{n_{k}}=\frac{g_{i} e^{-\left(E_{i} / k T\right)}}{g_{k} e^{-\left(E_{k} / k T\right)}}
$$

Radiation emitted from one atom in a plasma may be reabsorbed by others. The optical thickness of a plasma is defined as

$$
\tau(v)=\int K(v) \partial x
$$

where $K(v)$ is the frequency-dependent absorption coefficient per $\mathrm{cm}$ and $x$ is along the line of sight. In the case of an optically thick plasma, no radiation will be emitted. Providing that LTE holds, the absolute intensity spectrum resembles that of a blackbody radiator and the electron temperature can be determined from Planck's law:

$$
I_{\lambda}(T)=\frac{2 h c^{2}}{\lambda^{5}}\left[e^{h c / \lambda k T}-1\right]^{-1}
$$

The specific intensity, $I_{\lambda}(T)$, is defined, with respect to wavelength, as the radiant energy which penetrates a unit area in a unit time within a unit solid area perpendicular to the area. Rather,

$$
I_{\lambda} \equiv \lim \frac{\Delta E}{\Delta t \Delta A \Delta \Delta \Delta \lambda} \text { for } \Delta t, \Delta A, \Delta \Omega, \Delta \lambda+0
$$

In the case of an optically thin plasma, selfabsorption of radiation is neglected. Two different methods can be used to determine electron temperature.

Firstly, temperature can be determined from the relative intensities of two spectral lines of the same atomic species (e.g., two Al-I lines). The intensity ratio of the two lines is given by

$$
\frac{I}{I_{2}}=\frac{A_{1} g_{1} 2^{2} 2^{n} 1}{A_{2} g_{2} 1^{2} 1^{n} 2} \exp \left[\frac{-\left(E_{1}-E_{2}\right)}{k T}\right]
$$

\section{where}

indices 1 and 2 denote the spectral lines

$A$ is the transition probability

$g$ is the statistical weight

$z$ is the partition function

and

$n$ is the number density.

For two lines belonging to the same ionization stage, the partition functions $Z_{1}$ and $Z_{2}$ are the same. Also, in the ground state, $n_{2}=n_{1}$, so knowledge of number density is not required. Therefore, Eq. (12), becomes

$$
\begin{gathered}
\frac{I_{1}}{I_{2}}=\frac{A_{1} g_{1}{ }_{2}}{A_{2} g_{2}^{\lambda}} \exp \left[\frac{-\left(E_{1}-E_{2}\right)}{k T}\right] \\
\frac{\Delta T}{T}=\frac{k T}{\left(E_{1}-E_{2}\right)} \frac{\Delta\left(I_{1} / I_{2}\right)}{I_{1} / I_{2}}
\end{gathered}
$$

The accuracy of the temperature determination by the relative intensity method is 1 imited by the uncertainty in the value of the transition probabilities. However, accuracy may be improved by measuring a number of different lines. The range of the energy difference $\left(E_{1}-E_{2}\right)$ should also be as large as possible.

The absolute intensity method requires knowledge of not only transition probabilities but also plasma pressure. For an optically thin plasma, spectral intensity can be given in terms of the emission coefficient $\varepsilon_{v}$ over the optical path as

$$
I_{\nu}=\varepsilon_{\nu} \cdot x_{0}
$$

The absolute intensity, then, is

$$
I=\int I_{v} \partial v=x_{0} \int \varepsilon_{v} d v
$$

The emission coefficient of a spectral line can be defined by

$$
\varepsilon_{v}=\frac{h_{v}}{4 \pi} A_{k i} n_{k} P_{v}
$$

where

$A_{k i}$ is the transition probability for spontaneous emission

$\mathrm{nk}_{\mathrm{k}}$ is the population of atoms in the upper level $k$

$P_{v}$ is the normalized line profile.

$$
\text { Since } \int P_{v} d v \equiv 1 \text {, substitution of (17) into }
$$

(16) gives

$$
I=x_{0} \int \epsilon_{v} d v=\frac{h v}{4 \pi} A_{k i} n_{k} x_{0}=\frac{h v x_{0}}{4 \pi} A_{k i} n g_{j} \frac{e^{-E_{j} / k T}}{Z(T)}
$$

with $n \equiv P / k T$ where $P$ is the pressure.

The temperature obtained with this method is an average value, defined along the optical path of the

An Optical Multi-channel Analyzer (OMA) will be used to survey the plasma spectrum. Figure 12 gives a general illustration of the system. Light from a source (the plasma arc) traverses a slit and is diffracted by a holographic grating onto a detector consisting of an array of 512 silicon diodes. The induced charge on the detector is measured by an electron 
scanning technique and sent to the OMA for processing and analysis. The spectrum information can then be sent either to a teletype or plotter for print-out or to a memory disk for storage. The advantages of the OMA are its speed and its ability to display the entire spectrum in a single shot. Strong transition lines of CU-I and AI-I in the visible region are expected to be observed in the rail accelerator plasma armature. One good calibration procedure for the spectros-

copic technique involves the observation of a standard light source of known intensity with the instruments to be calibrated. In this manner, all system components, e.g., detectors and recorders, are taken into account collectively.

Blackbody radiators make excellent calibration sources as they are characterized in Planck's law by a single parameter, the temperature. However, in usual laboratory practice, a secondary intensity standard is used. The tungsten ribbon 1 amp is the most common intensity standard for the visible region. For specific wavelength, the brightness temperature is given as a function of the filament current. This temperature can then be used to calculate the spectral intensity emitted at this wavelength, using known radiation constants. Because the tungsten ribbon lamp is only calibrated at one wavelength, uncertainties in temperature can arise due to the optical properties of the lamp window (reflection losses) and the filament's emissivity dependence on wavelength. Nevertheless, for wavelengths above $3000 \AA$, it should be possible to obtain accuracy of the intensity standard to about one
percent. 17

\section{High Speed Framing and Streak Camera Photography}

Visual observation of the plasma arc accelerations is made possible with an image converter camera. In the camera, light energy radiated from the plasma arc is focused on the image converter tube photo cathode which converts the photon image to an electron image. This electron image is then accelerated and focused with a brightness gain on the photoanode of the tube. A precision recording camera provides the photoanode-to-film optical system.

Two operation modes are available with camera plug-in units. A standard framing unit permits the recording of spatial changes of the plasma. Adjustable exposure times from 50-200 nanoseconds are available with frame intervals of 0.5-10 nanoseconds. Two streak units enable measurement of the time rate of change of the plasma. A wide range unit allows exposure times of $0.5,1,2,5$, and $10 \mathrm{msec}$ with a film writing rate of $100,50,25,10$, and $5 \mathrm{~mm} / \mathrm{usec}$, respectively. The other streak unit is designed for high speed; it has available film writing rates of $2500,1000,500$, and $250 \mathrm{~mm} / \mathrm{usec}$ for exposure times of $20,50,100$, and $200 \mathrm{nsec}$

\section{Current Measurements}

Current transformers give accurate, reliable measurements of circuit current, have a well developed technology, and are commercially available. They provide a low noise pulse with little interference from associated ground loops. However, limitations imposed by the design constraints of the transformer must be taken into account. One important factor affecting accuracy is the risetime limitation on the transformer due to the geometry of the primary arcuit (inductance effects). Another limitation is that of total charge $(I-t)$ which may constrain either peak current or pulse length. 18

A Pearson Model 2093 current transformer is mounted on the transition piece between the header assembly and the inductance coil. It has an output of $1 \mathrm{mV} / \mathrm{A}$, a useable risetime of 2 manoseconds, and a rated $1 \cdot t$ of 1200 Asec. The current transformer signal is reduced by a $100 \mathrm{x}$ high voltage probe at the oscillosocope.

A Rogowski coil is a current transformer in which the secondary is a toroidally wound coil through which the measured current passes. See Fig. 13. The secondary senses the magnetic field generated by the main current. From a combination of the Biot-Savart Law and Faraday's Law of Induction, the voltage across terminals $a, b$ can be written as

$$
V_{a, b}=n A \frac{d B}{d t}=2 \times 10^{-7} \frac{n A}{\bar{T}} \frac{d I}{d t}
$$

where

$n$ is the number of toroidal turns

$A$ is the area of a single turn

$T$ is the major radius

$\mathrm{dI} / \mathrm{dt}$ is the time derivative of the current

Inserting an $R C$ integrating circuit between the Rogowski coil and the oscillosocope gives an output

$$
v_{0}=\frac{1}{R C} \int v_{a, b} d t=\frac{\left(2 \times 10^{-7}\right) n A I}{r R C}
$$

The time constant RC of the integrator should be at least an order of magnitude longer than the pulse length to minimize capacitive effects.19,20 Rogowski coils for the LeRC experiments were calibrated against the Pearson current transformer in a series of inductive load tests. A single bank module was discharged into a low resistance, inductive load $(3.7 \mu \mathrm{H})$ giving current pulses from $30 \mathrm{kA}$ to $120 \mathrm{kA}$. Because the output signal is integrated over a closed loop, measurements are independent of current density. Rogowski coils are ideal for measurements in physically Timited spaces such as the coax input to the rail accelerator.

\section{Voltage Measurements}

Divider networks were designed to measure the large, rapidly varying breech voltage and the relatively constant muzzle voltage of the accelerator. The networks consist of a series of low inductance carbon resistors used in combination with a small current transformer (Pearson 4100: output $=1 \mathrm{~V} / \mathrm{A}$, I $\cdot t=0.002 \mathrm{Asec}$ ). The breech divider is connected to the fuse holder at the bolts closest to the accelerator. The connecting leads are twisted to further minimize inductance. The muzzle divider measurement leads are held by screws to the rail stubs, close to the $\mathrm{G}-10$ support structure.

\section{Projectile Performance Measurements}

Two different techniques are used to obtain information on projectile performance. A set of $\mathrm{dB} / \mathrm{dt}$ probes stationed every $20 \mathrm{~cm}$ along the length of the accelerator is used to establish the projectile's position as a function of time while in the bore. Each probe is a magnetic flux coil consisting of a number of wire turns wound on a circular magnetic rod. The axis of each coil is positioned parallel with the accelerator bore so that the coil detects primarily the field associated with the plasma arc. Each coil produces a voltage proportional to the time rate of change of the magnetic field. As the arc and the projectile pass the dB/dt station, the flux in the coil increases. The wire turns of each successive $\mathrm{dB} / \mathrm{dt}$ station are wound in opposite directions. The zero crossings on the probe voltage output, then, indicate that the center arc is directly in line with the
station. 6,21 
A simple timing device is used to obtain projectile velocity during free-flight. The timing device consists of two screens electrically connected to a voltage box and digital timer. Each screen is made up of two isolated foil sheets separated with a piece of insulation. A battery maintains a potential drop across the foil sheets of 450 volts. When the projectile pierces the first screen foil edges are bent from the leading foil, through the insulator, so that they touch the backing foil. Thus, the battery circuit is shorted and a subsequent pulse is sent to the digital timer. The projectile then pierces the second screen sending another pulse. The time of flight interval between shorting the two screens gives projectile velocity.

\section{Rail Displacement Measurements}

Optical sensors can be used to measure the distance to a reflecting surface. One method is to use bundles of parallel optical fibers as shown in Fig. 14(a). Half of the bundle is used to transmit light to the sensing end while the other half of the fibers serve as receiving elements. When the sensor end is touching the surface to be measured, no light can be reflected back into the receiving fibers. As the surface is displaced away from the sensor end, transmitted light will reflect off the surface and be detected by the receivers. The intensity of the received light varies linearly with surface displacement up to a distance defined by the probe geometry. Figure 14(b) gives a typical response curve of the fiber optic probe. At a certain displacement the entire surface of the receiver is illuminated by the reflected cone of light. At this point, the response curve of the sensor reaches its peak and is entirely a function of surface reflectivity. As displacement increases further, receiver illumination decreases in approximately inverse proportion to the square of the displacement.

A steep response curve can be obtained with a distribution of transmitting and receiving fibers, enabling precise measurement of small displacements, such as rail movement during the acceleration process. Another advantage of using an optical link to measure rail displacement is that it allows the electronics to be placed far away from the electrostatic and magnetic fields present in the accelerator system. 22,23 The high level energy input to the rail accelerator may cause a shock displacement of the entire gun assembly. In order to compensate for this movement, a technique using two optical probes is employed. Figure 15 shows the mounting of two optical sensors on the $4 \times 6 \mathrm{~mm}$ bore railgun assembly.

The probes are held fast in micrometer heads such that their direction is perpendicular to the rail motion. Deflection from the individual sensors are added to get a signal which is proportional to the total motion of the rails. Because the probes view the outer face of the rails, little optical interference from the disturbance of the plasma passing by is expected.

The two micrometer heads are held rigid with respect to one another by a heavy yoke assembly, as shown in the figure. Flexure supports, mounted on the neutral axes of the accelerator, absorb any shock displacement of the railgun structure.

\section{Summary}

An in-house research program at LeRC has been initiated to investigate the technical feasibility of using rail accelerators for space propulsion applications. Two different types of rail accelerators were fabricated with the objectives of demonstrating projectile velocities of $15 \mathrm{~km} / \mathrm{sec}$ and characterizing the plasma armature with a basic arc model. This paper presented a study of available diagnostic techniques and their application to the rail accelerator experiments.

\section{References}

1. E. E. Rice, L. A. Miller, and R. W. Earhart, "Preliminary Feasibility Assessment for Earth-to-Space Electromagnetic (Rail Gun) Launchers," NASA CR-167886, June 1982.

2. Private communication with Prof. Alan Larson, Georgia Institute of Technology.

3. Private communication with Charies J. Michels, NASA-LERC, retired.

4. J. P. Barber and H. F. Swift, Modern Electric Rail Gun Technology" in ICIASF'79 Record. New York: Institute of Electrical and Electronic Engineers, pp. 141-145, 1979.

5. D. P. Bauer, T. J. McCormick, and J. P. Barber "Electric Rail Gun Projectile Acceleration to High Velocity," in AIAA, Japan Society for Aeronautical and Space Sciences and OGLR' I6th International Electric Propulsion Conference, New Or leans, AIAA Paper 82-1939, Nov. 1982.

6. D. P. Bauer, T. J. McCormick, and J. P., "Electric Rail Launcher System Design and Test Evaluation," IAP Research, Inc., IAP-TR-82-6, Nov. 1982.

7. S. T. Gooder, "Electromagnetic Propulsion Test Facility," to be published.

8. J. F. Kerrisk, "Current Distribution and Inductance Calculations for Rail-Gun Conductors, "Los Alamos National Laboratory, LA-9092-MS, 1981.

9. T. J. Tucker and R. P. Toth, "EBW1: A Computer Code for the Prediction of the Behavior of Electrical Circuits Containing Exploding Wire Elements," Sandia Laboratories, SAND-75-0041, Apri 1 ments,"

10. I. R. McNab, "Electromagnetic Macroparticle Acceleration by a High Pressure Plasma," J. Appl. Phys. vol. 51, pp. 2549-2551, May 1980.

11. J. D. Poweli and J. H. Batteh, "Plasma Dynamics of an Arc-Driven, Electromagnetic, Projectile Accelerator," J. Appl. Phys., vol. 52, pp. 2717-2730, Apr. 1981

12. Powe11, J. D., "Two Dimensional Model for Arc Dynamics in the Rail Gun," Army Armament Research and Development Command, Aberdeen Proving Ground, Md. Ballistic Research Lab., ARBRL-TR-02423, 0ct. 82.

13. Y. C. Thio, "PARA: A Computer Simulation Code for Plasma Driven Electromagnetic Launchers," Aug. 1982, unpublished report.

14. F. C. Jahoda, "Lasers as a Tool for Plasma Diagnostics," Los Alamos National Laboratory LA-UR-81-896, 1981

15. F. C. Jahoda and G. A. Sawyer, Optical Refractivity of Piasmas," in Plasma Physics, Part B, H. R. Griem and R. H. Lovberg, eds. New York: Academic Press, 1971, pp. 1-48.

16. W. Lochte-Holtgreven, ed., Plasma Diagnostics. Amsterdam: North-Holl and Pub. Co., 1968.

17. H. R. Griem, Plasma Spectroscopy. New York: McGraw-Hill Book Company, 1964.

18. M. E. Wilmer and P. A. Pearson, "Precise Measurement of Current in Puised Power Systems, "in Measurement of Electrical Quantities in Pulse-Power Systems, R. H. McKnight and R. E. Hebner, Jr., eds., NBS-SP-628, pp. 194-203, June 1982.

19. M. S. DiCapua, "Rogowski Coils Fluxmeters, and Resistors for Pulsed Current Measurments" in Measurement of Electrical Quantities in Pulse-Power systems, R. H. McKnight and R. E. Hebner, Jr., ed., NBS-SP-628, pp. 175-193, June 1982.

20. S. Glasstone and R. H. Lovberg, "Plasma Diagnostic Techniques" in Controlled Thermonuclear Reactions; an introduction to theory and experiment $4,1960$. 
21. K. A. Jamison and H. 5. Burden, "Arc Armature Diagnost ic Experiments on an Electromagnetic Gun, " Arradcom Technical Conference, July 1982.

22. C. Menadier, C. Kissinger, and $M$. Adkins, "The Fotonic Sensor," Bulletin No. 02-100, Mechanical Technology, Inc.

23. D. H. Locke, "Lithographic Plate Surface Inspection Using Fiber Optics, Mechnical Technology, Inc. 


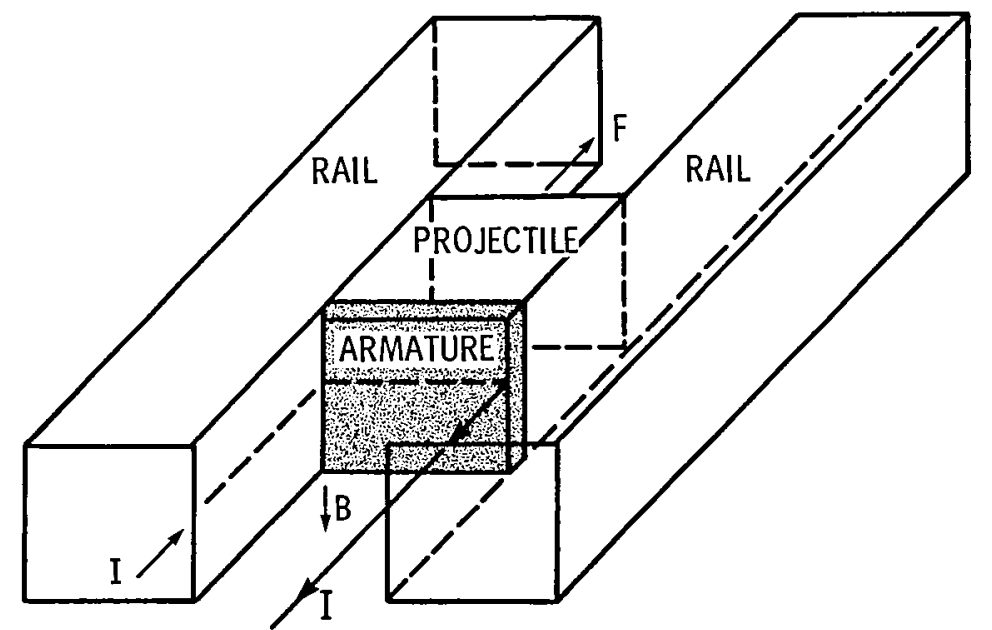

Figure 1. - Rail accelerator principle.

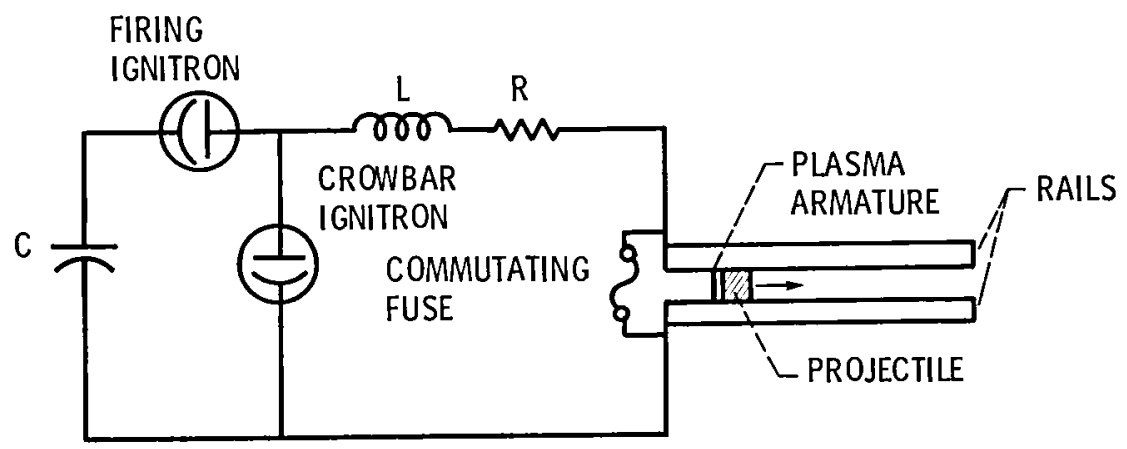

Figure 2 - Electrical schematic for pulsed power energy system. 


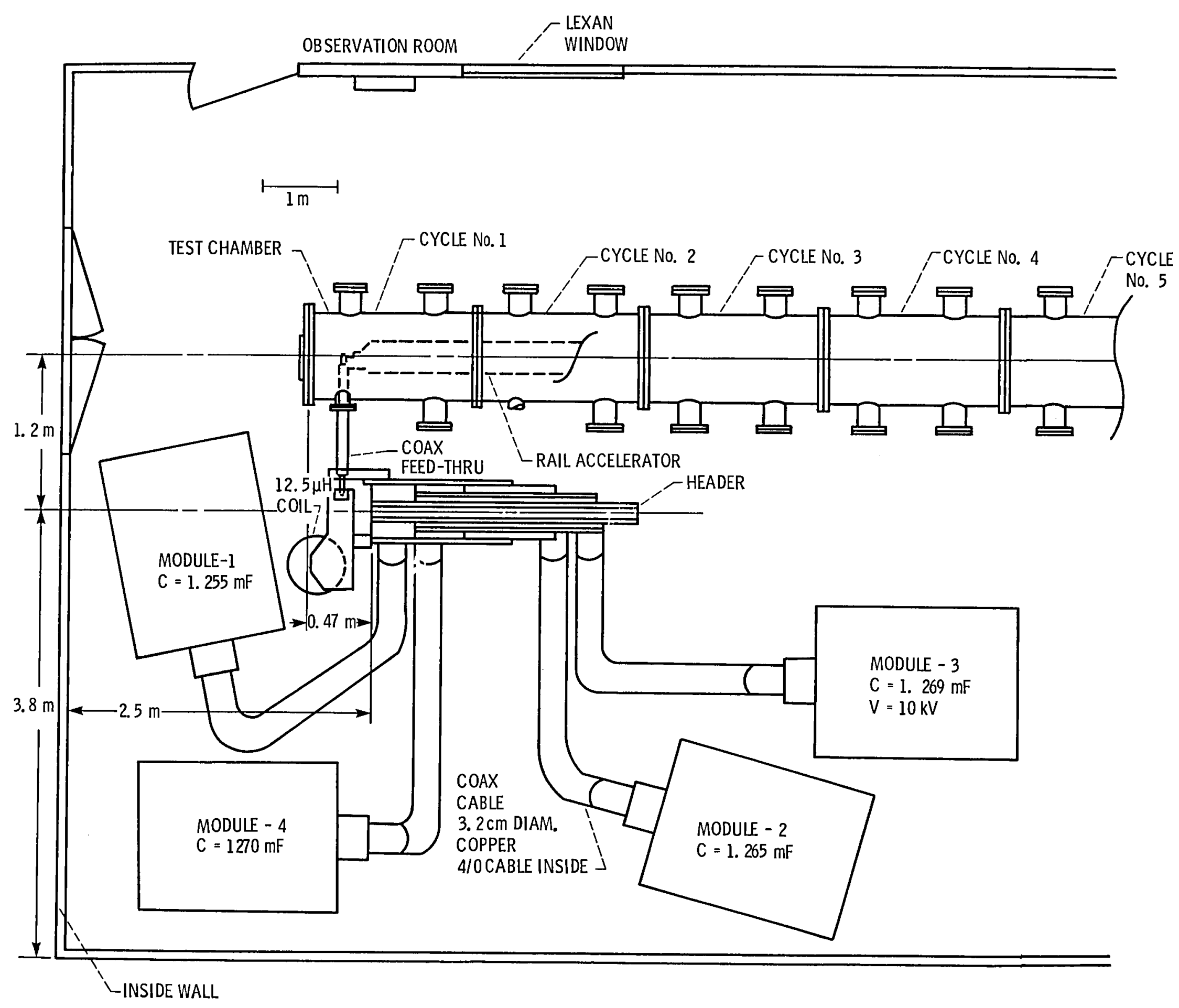

(a) $240 \mathrm{~kJ}$ pulsed power system.

Figure 3. - LeRC rail accelerator facility. 


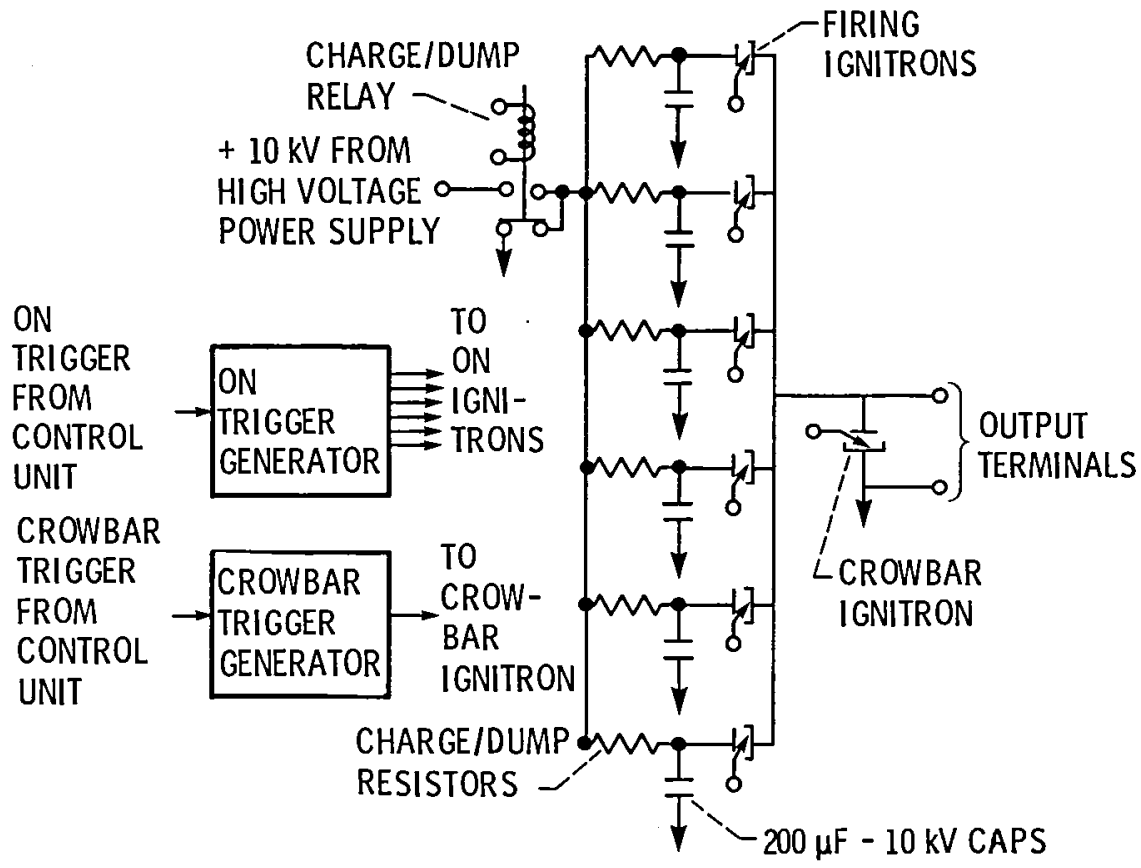

(b) Simplified schematic diagram for one $60 \mathrm{kJoule}$ module.

Figure 3. - Concluded.

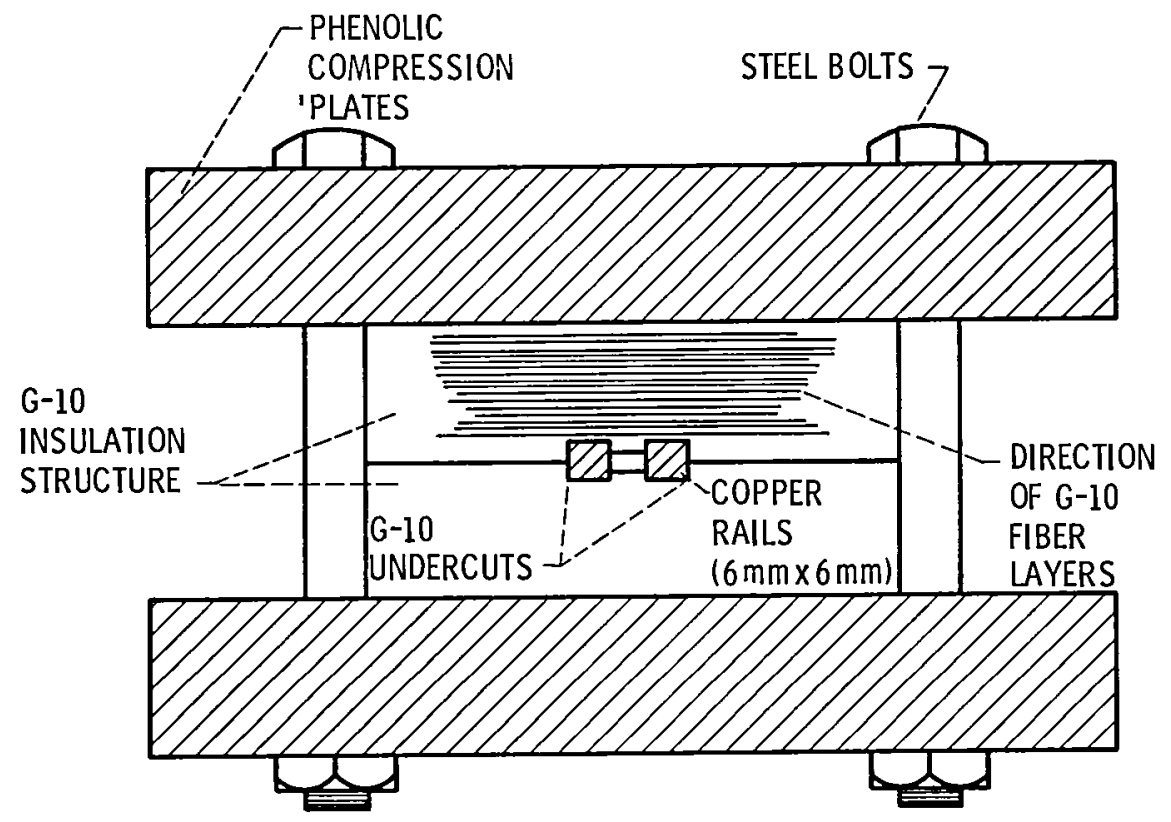

Figure 4. Three-meter long - $4 \times 6 \mathrm{~mm}$ bore accelerator tested at LLNL. Direction of $\mathrm{G}-10$ fiber layers is in the horizontal plane. 
PHENOLIC

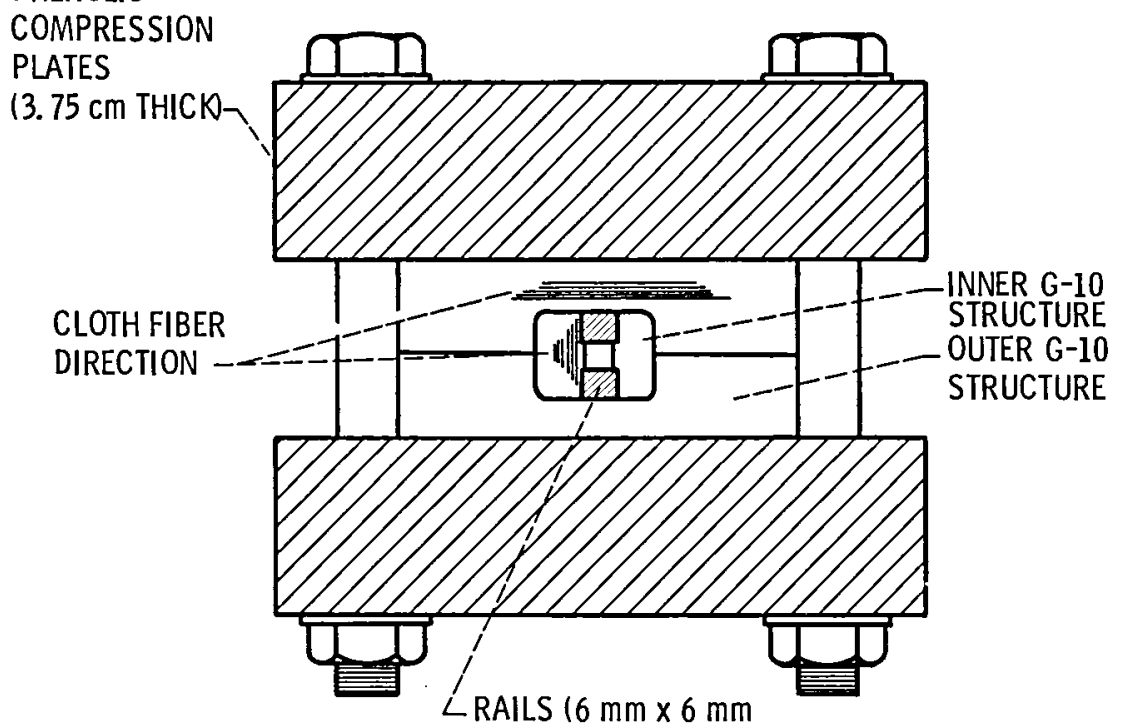

Figure 5. - One meter long $-4 \times 6 \mathrm{~mm}$ bore rail accelerator. Clamping action is against the outward rail forces.

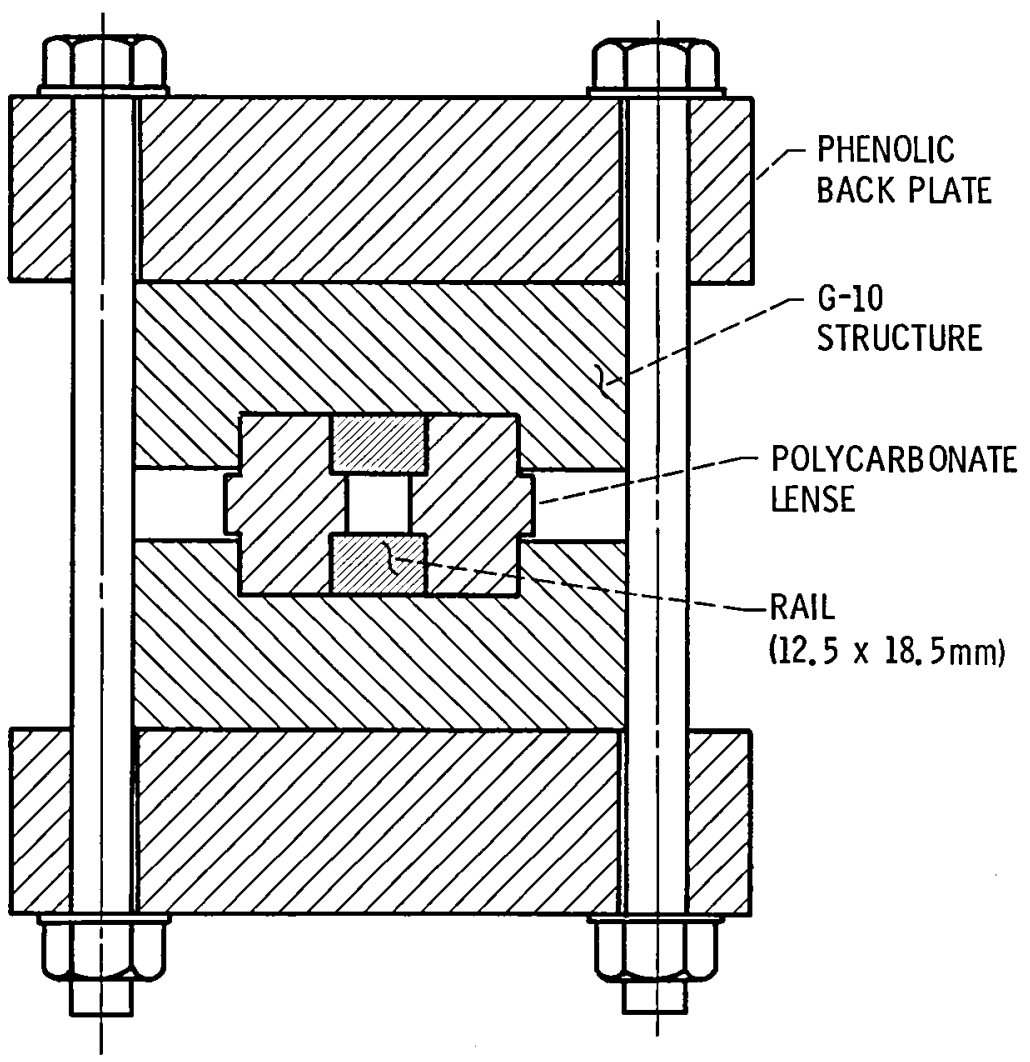

Figure 6. - One meter long - $12.5 \times 12.5 \mathrm{~mm}$ bore rail accelerator. Clear polycarbonate sidewalls permit observation of plasma arc acceleration. 


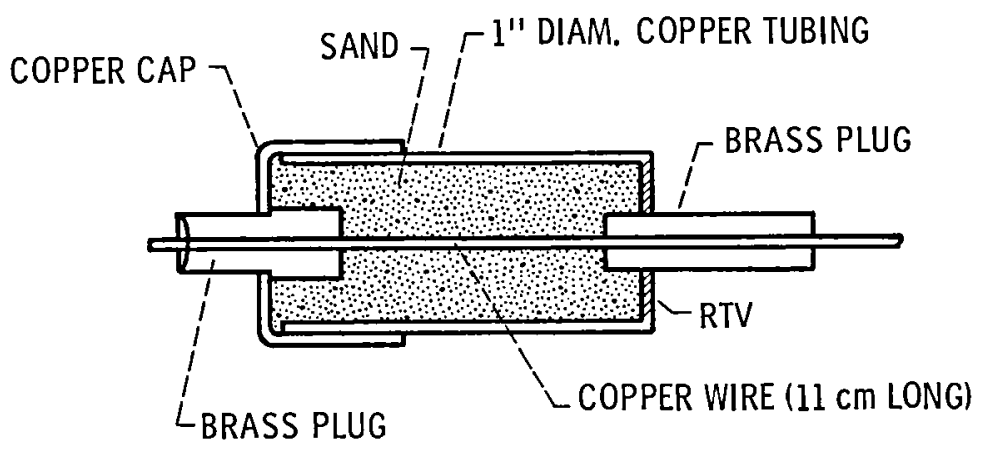

(a) Commutating fuse.

Figure 7. - Commutating fuse and holder.

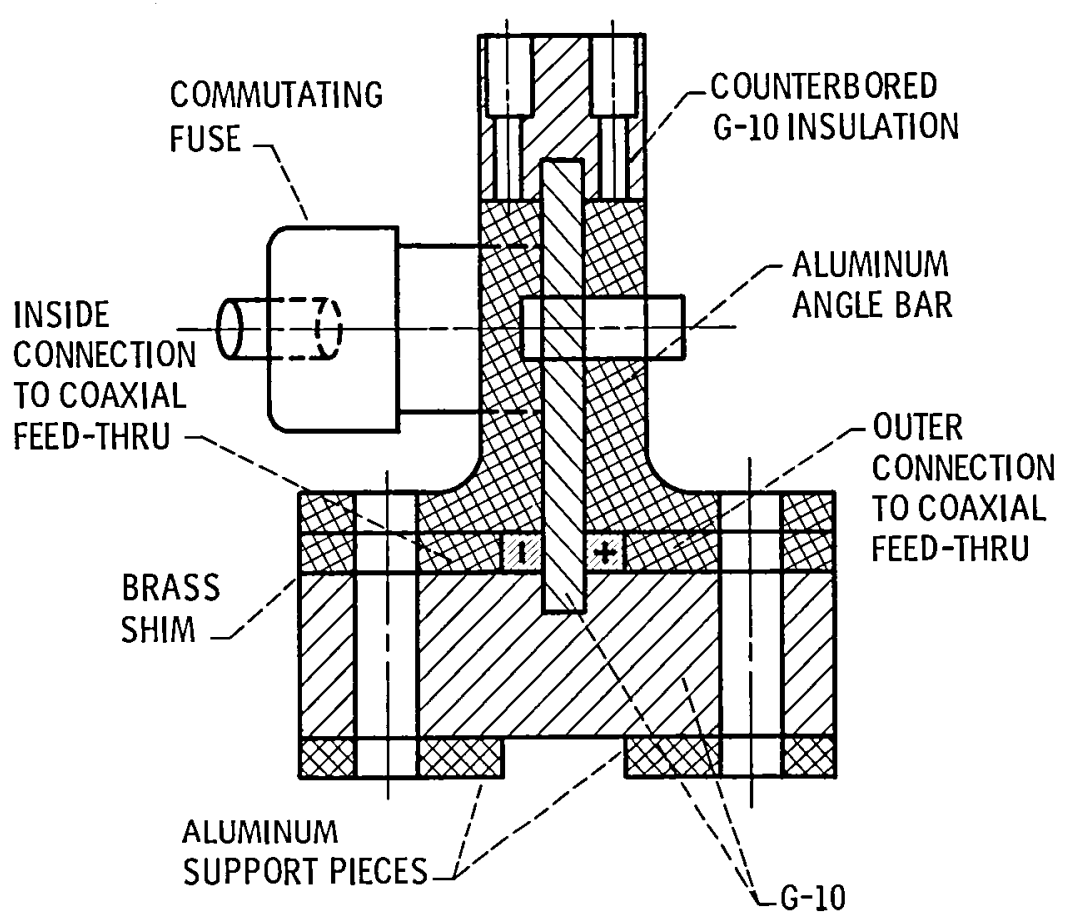

(b) Fuse holder side view.

Figure 7. - Concluded. 


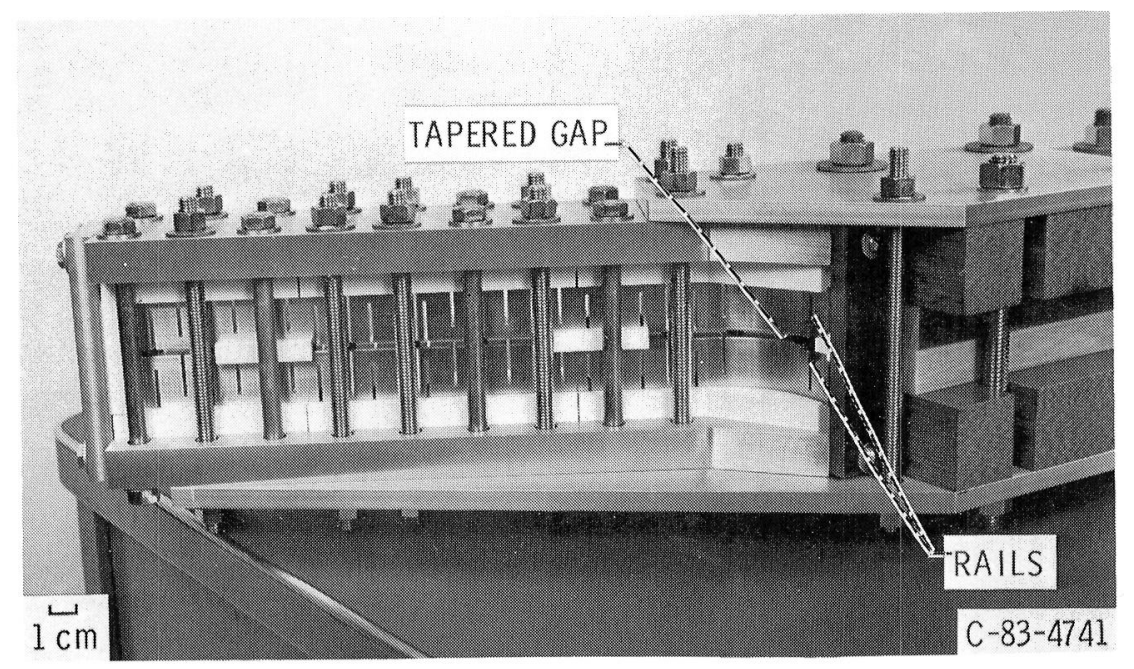

Figure 8. - Muzzle supressor.

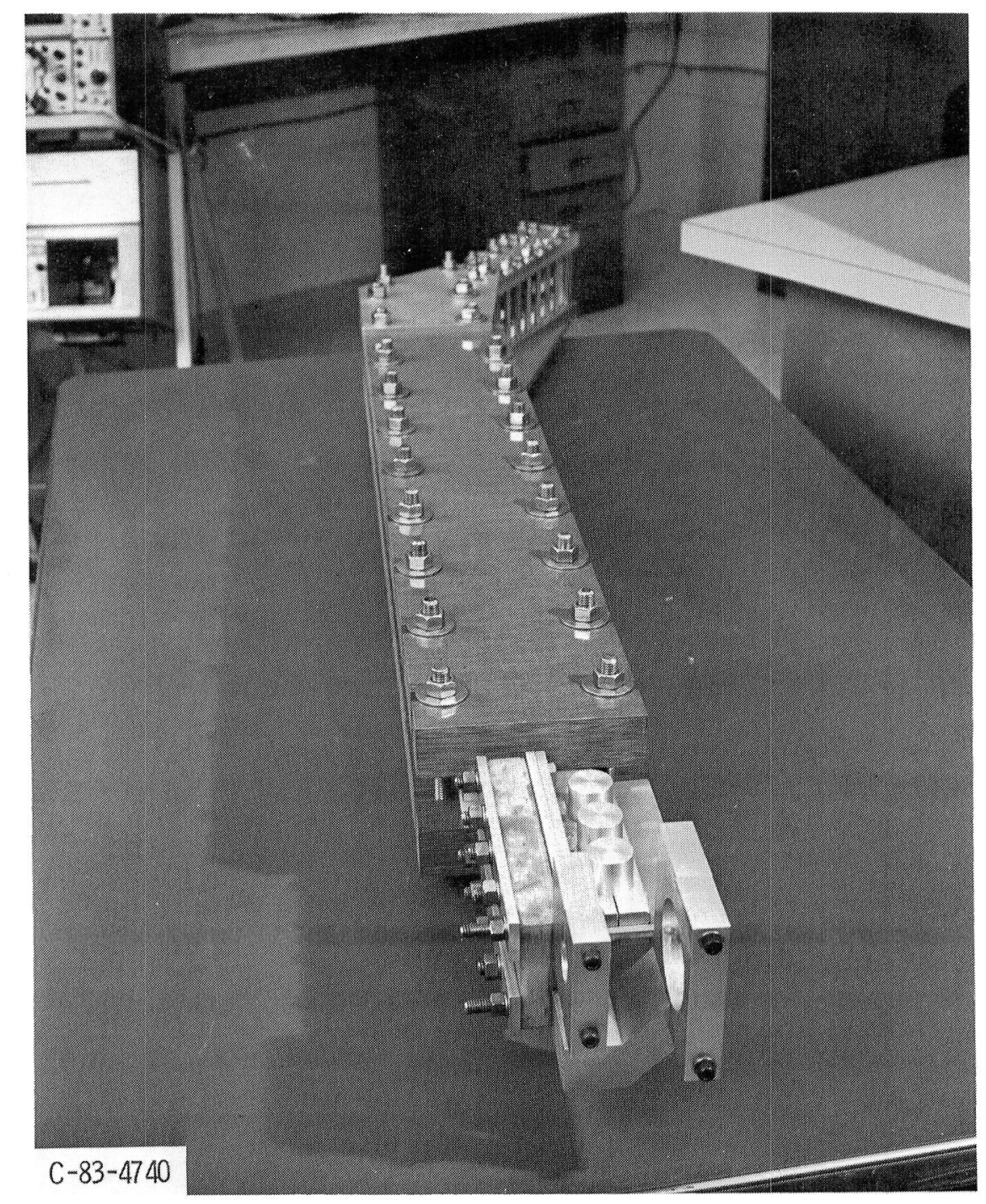

Figure 9. - 1 meter, $4 \times 6 \mathrm{~mm}$ bore rail accelerator assembly. 


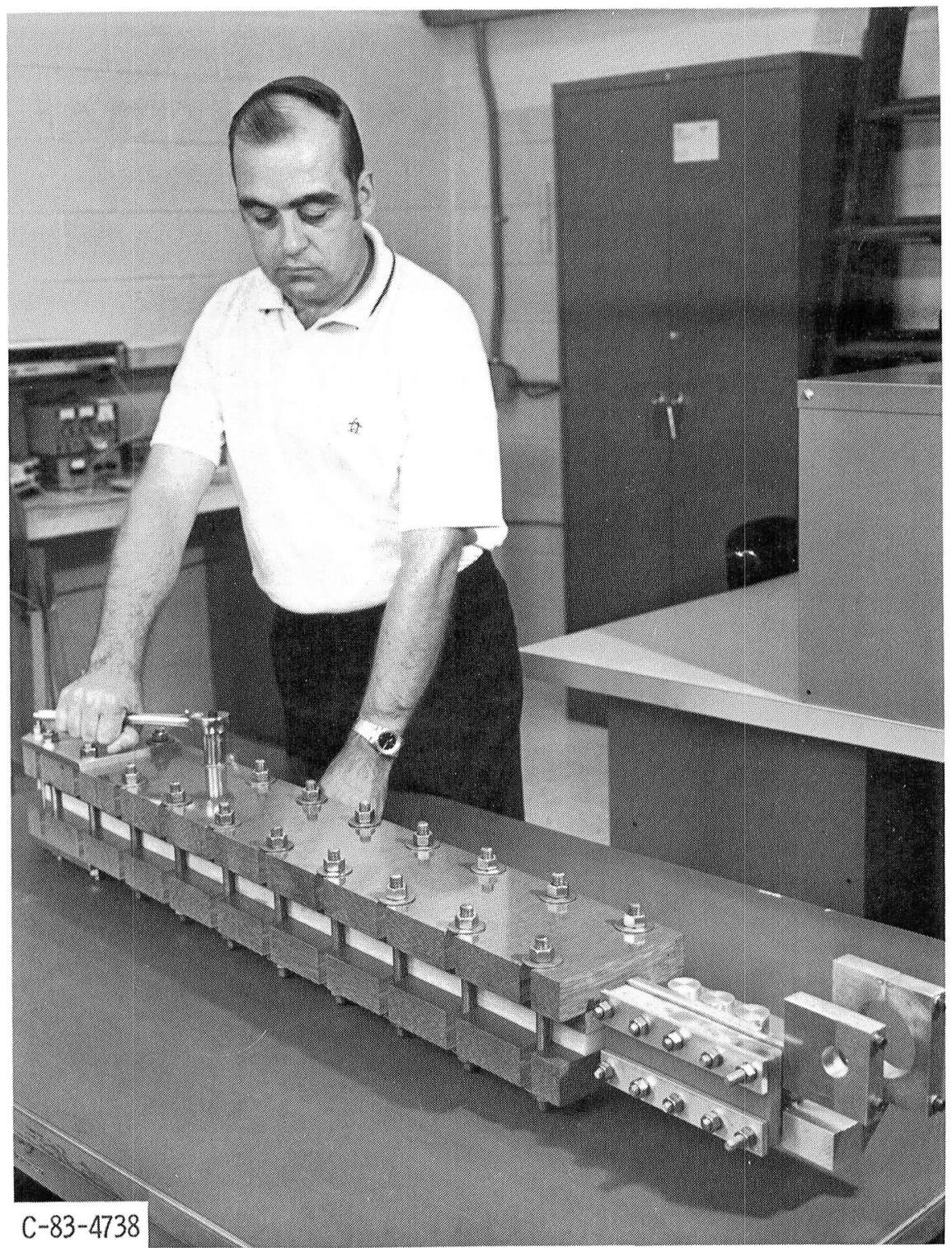

Figure 9. - Concluded.

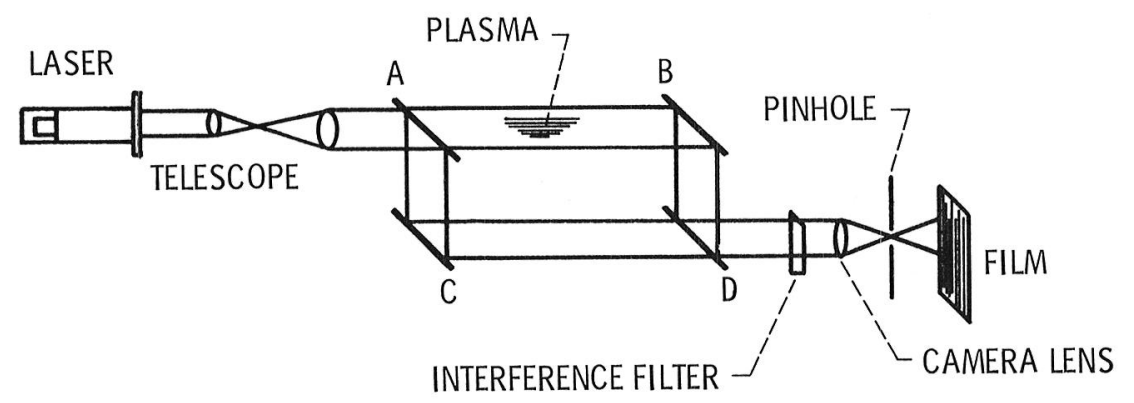

Figure 10. - Mach-Zehnder interferometor. 


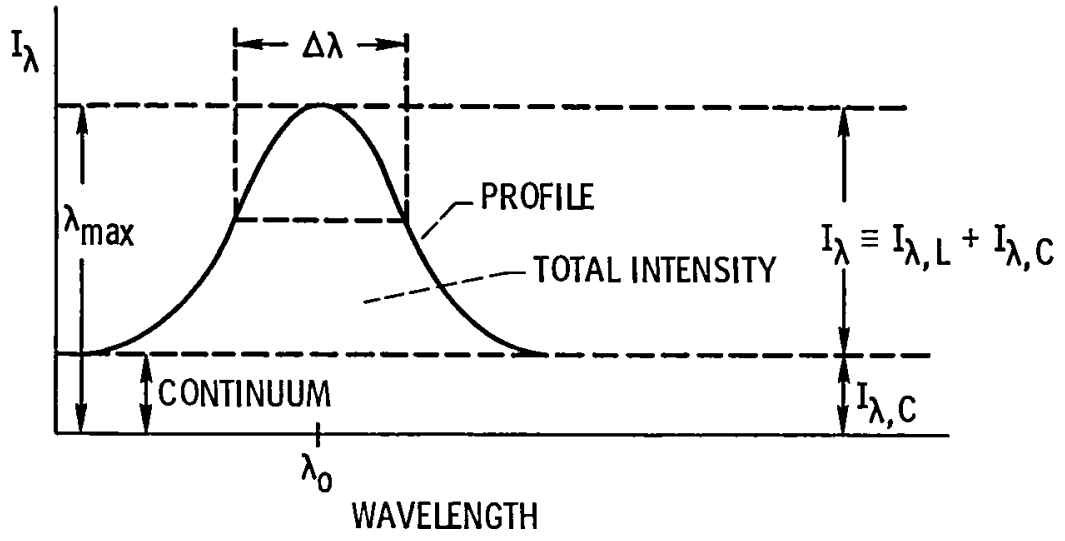

Figure 11. - Information contained in a spectrum (specific intensity versus wavelength).

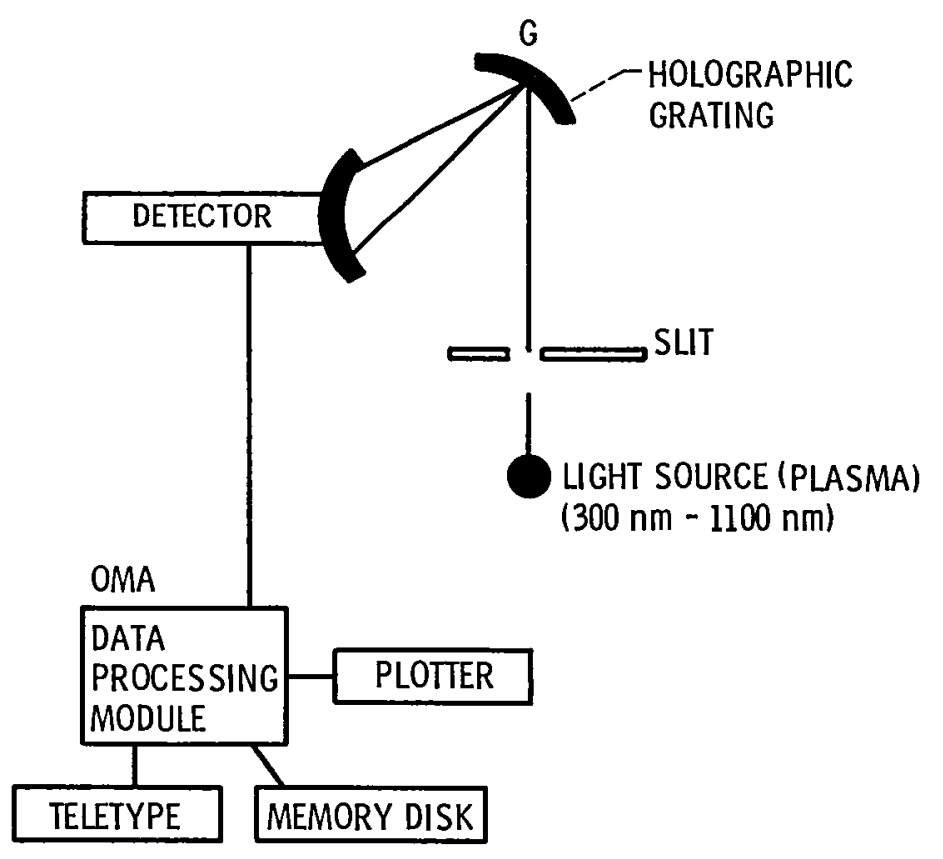

Figure 12 - Optical multichannel analyzer. 


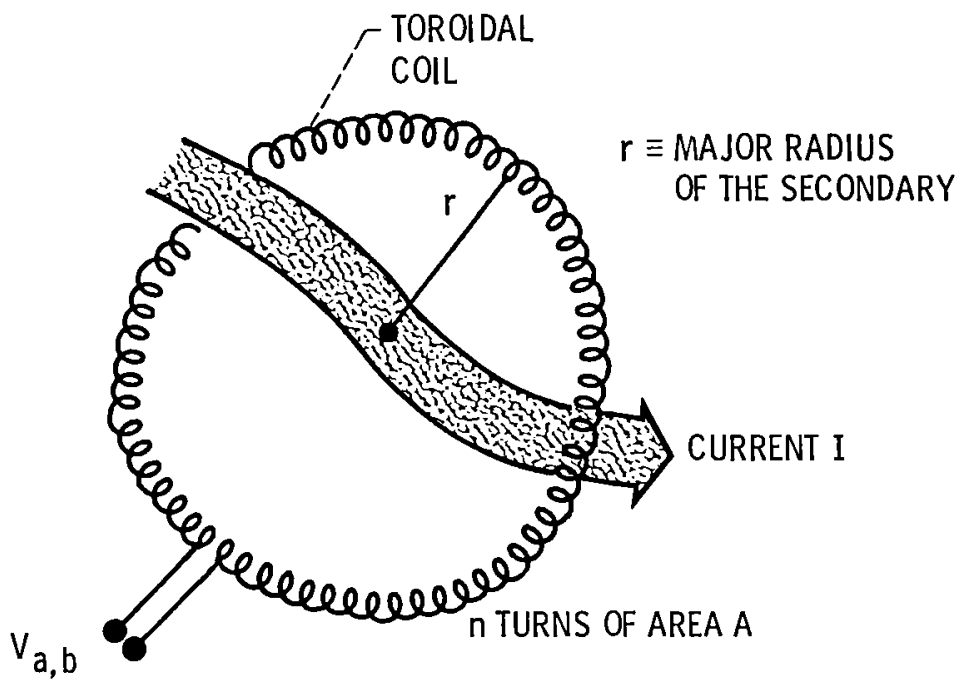

Figure 13. - Rogowski coil. 


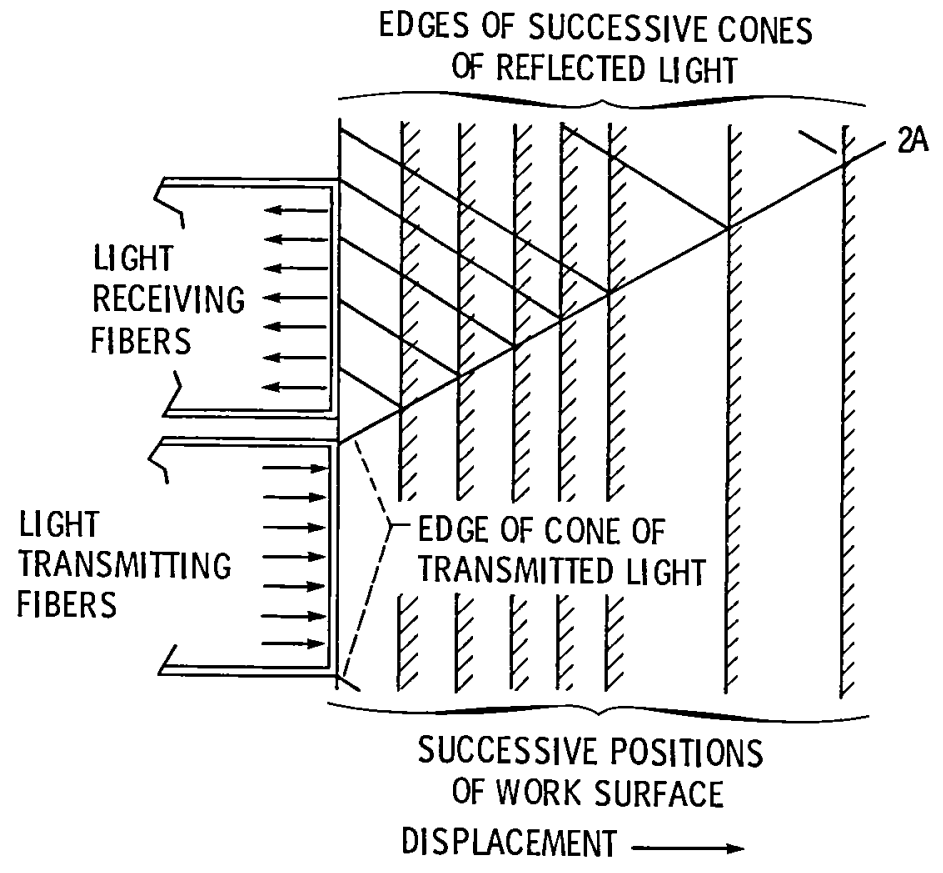

(a) Optical displacement principle.

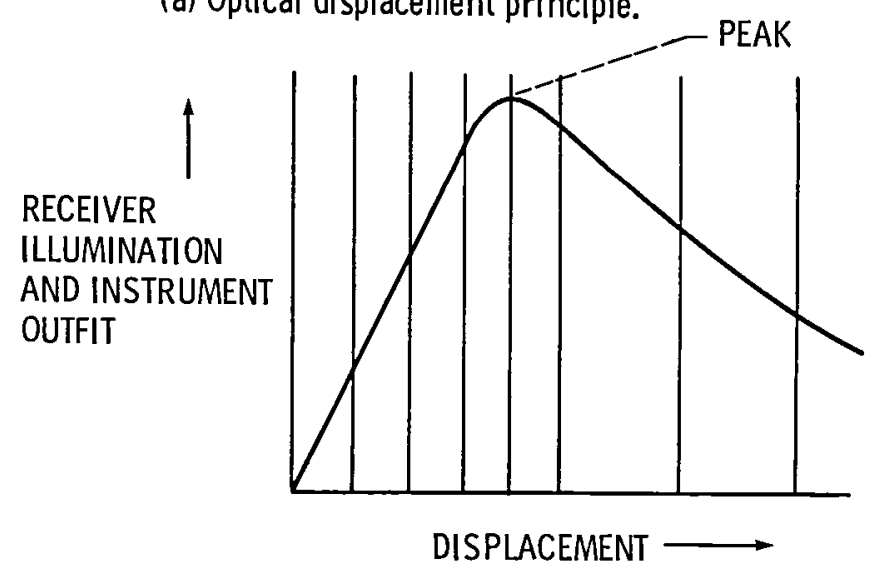

(b) Response curve.

Figure 14. - Fiber optic probe (displacement measurements). 


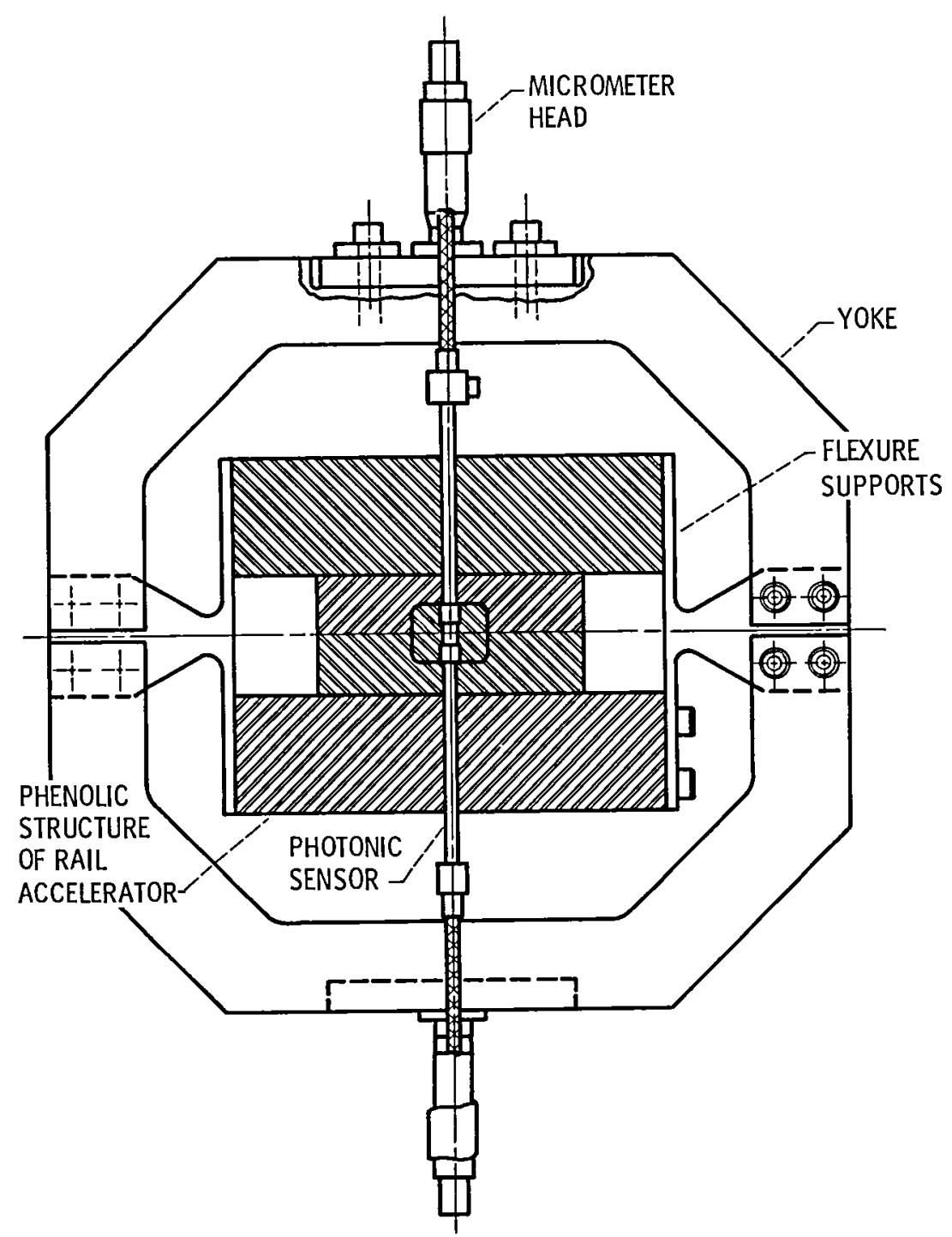

Figure 15. - Mounting of two optical sensors in 1 meter $-4 \times 6 \mathrm{~mm}$ accelerator. 


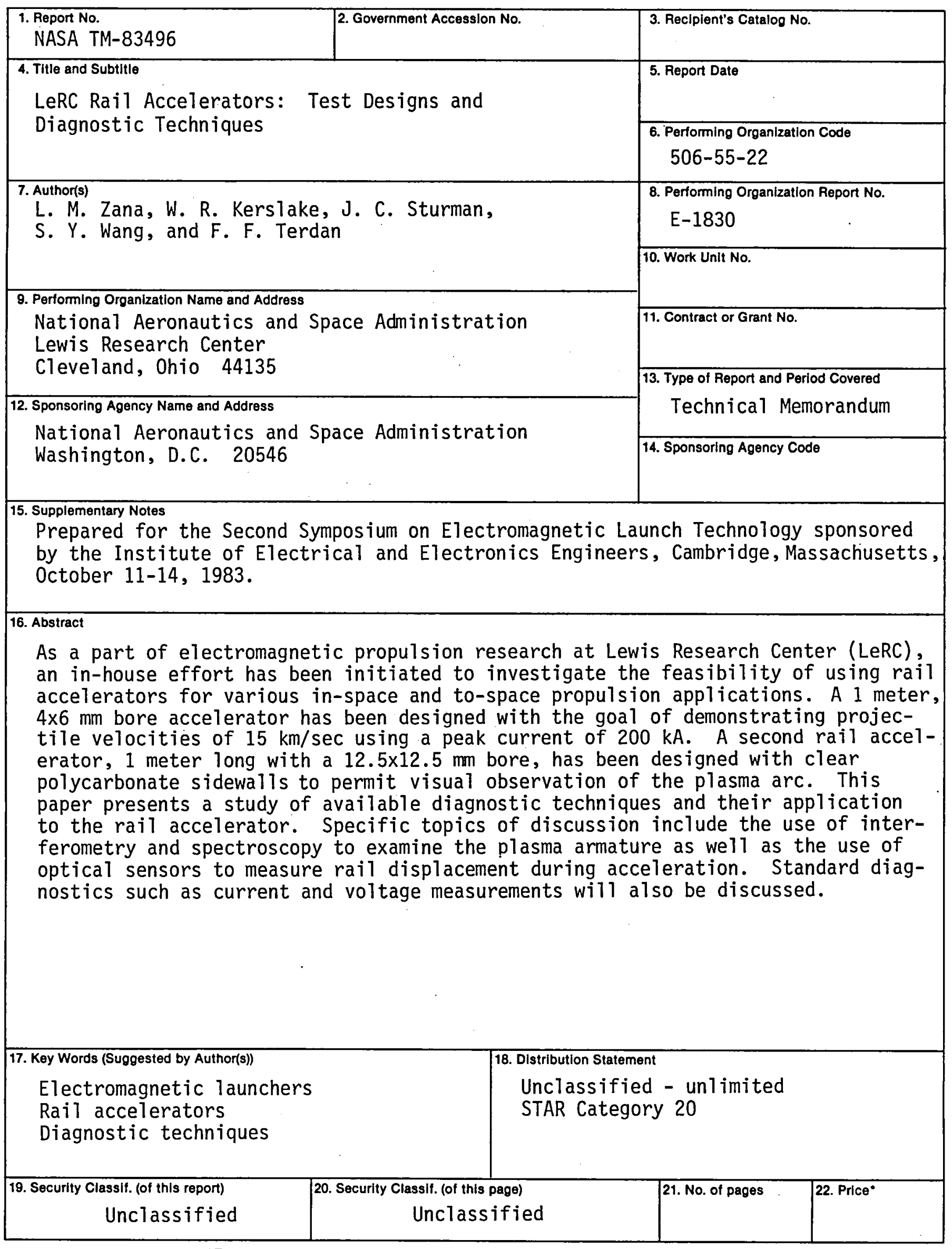

"For sale by the National Technical Information Service, Springfield, Virginia 22161 
National Aeronautics and Space Administration

Washington, D.C.

20546

Official Business

Penalty for Private Use, $\$ 300$
SPECIAL FOURTH CLASS MAIL BOOK

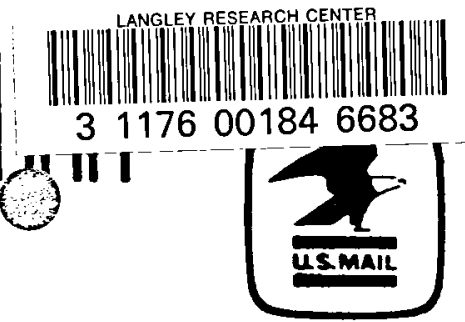

Postage and Fees Paid

National Aeronautics and

Space Administration

NASA 451 